\title{
The use of assisted reproductive technology before male factor infertility evaluation
}

\author{
Madhur Nayan ${ }^{1}$, Nahid Punjani ${ }^{2}$, Ethan Grober ${ }^{1}$, Kirk Lo ${ }^{1,3,4}$, Keith Jarvi ${ }^{1,3,4}$ \\ ${ }^{1}$ Division of Urology, Department of Surgery, University of Toronto, Toronto, Canada; ${ }^{2}$ Division of Urology, London Health Sciences Centre, \\ Western University, London, Canada; ${ }^{3}$ Lunenfeld Tannenbaum Research Institute, Mount Sinai Hospital, Toronto, Canada; ${ }^{4}$ Faculty of Medicine, \\ Institute of Medical Science, University of Toronto, Toronto, Canada \\ Contributions: (I) Conception and design: All authors; (II) Administrative support: K Jarvi; (III) Provision of study material or patients: E Grober, K \\ Lo, K Jarvi; (IV) Collection and assembly of data: All authors; (V) Data analysis and interpretation: All authors; (VI) Manuscript writing: All authors; \\ (VII) Final approval of manuscript: All authors. \\ Correspondence to: Madhur Nayan. Division of Urology, Department of Surgery, University of Toronto, 610 University Ave, Toronto, Ontario M5G \\ 2M9, Canada. Email: madhur.nayan@mail.utoronto.ca.
}

Background: Some centers offer assisted reproductive technologies (ARTs) [intra-uterine insemination (IUI) and in-vitro fertilization (IVF)], to treat certain couples with male factor infertility without having the men assessed by male infertility specialists. We sought to compare characteristics of couples having or not having prior ART use.

Methods: We used our prospectively collected database to identify men undergoing an initial evaluation for male infertility between 1995-2017. We obtained data on patient demographics, use of IUI and IVF, and semen analysis parameters. We used multivariable logistic regression to identify characteristics associated with prior use of ART.

Results: One thousand and five hundred forty-five out of 8,962 (17.2\%) men reported use of ARTs prior to evaluation. Of these, 258 tried both IUI and IVF. More than one attempt was reported in $470(37.2 \%)$ and 154 (28.2\%) of men with prior IUI and IVF, respectively. Younger male age [adjusted odds ratio (aOR) $0.97 /$ year; $95 \%$ confidence interval (CI), 0.95 to 0.99], older female partner age (aOR 1.07/year; 95\% CI, 1.04 to 1.10), and year of visit (aOR 1.05/year; 95\% CI, 1.01 to 1.09) were significantly associated with prior IUI. Older female partner age (aOR 1.07/year; 95\% CI, 1.02 to 1.12) was significantly associated with prior IVF, but not male age or year of visit. Semen analysis parameters were not associated with prior ART.

Conclusions: The prior use of ART is common among men presenting for an initial evaluation at a male infertility specialty clinic. Older female partner age was associated with use of reproductive technologies prior to evaluation, however, semen analysis parameters were not.

Keywords: Artificial insemination; fertilization in vitro; infertility male; reproductive technologies; urology

Submitted Jun 10, 2018. Accepted for publication Jun 11, 2018.

doi: $10.21037 /$ tau.2018.06.08

View this article at: http://dx.doi.org/10.21037/tau.2018.06.08

\section{Introduction}

Infertility is a global problem and it has been estimated that in $2010,1.9 \%$ of child-seeking women aged $20-44$ were unable to have a first live birth within 5 years, and $10.5 \%$ of child-seeking women who had at least one birth were unable to have another child (1). It has been shown that a male factor is the only cause of infertility in approximately $20 \%$ of couples trying to conceive, and contributes to another $40 \%$ of infertility cases (2).

Male factor infertility is typically investigated by urologists who investigate, provide management options and counsel couples with infertility. The management options include treating identified male infertility factors or/and using 
assisted reproductive technologies (ARTs) such as intrauterine insemination (IUI) and in-vitro fertilization (IVF). The success rate of these technologies depends on various factors, including semen quality (3-5). Despite initial failure, many patients using these technologies often undergo repeated attempts, resulting in increased treatment burden and costs. Given that these treatments are expensive (6), it is important to use them judiciously. The male fertility specialist may have a role in counselling a patient on their chance of success with the use of reproductive technology, particularly in the context of multiple attempts, as well as potentially optimizing a patient's semen parameters.

To date, however, there is no data on the use of ARTs before male fertility factor evaluation, the clinical characteristics of these couples and the semen quality of these patients. Therefore, the objective of this study was to evaluate characteristics associated with use of ARTs prior to evaluation at a male fertility subspecialty clinic.

\section{Methods}

After obtaining approval from the Research Ethics Board, we identified men presenting for an initial evaluation at a male fertility subspecialty clinic at a tertiary academic center between 1995 and 2017 using our prospectively collected database. Prior to consultation, patients completed a detailed questionnaire regarding demographics, medical history, partner information, and use of infertility treatments. All of the reported answers were confirmed by the interviewing physician.

From the database, we abstracted information on patient age, duration of infertility, coital frequency, year of consultation, partner age, medication use, cigarette smoking history, use of marijuana, and use of ARTs prior to evaluation. We also obtained data on the first semen analysis performed after consultation at our clinic. Specific semen parameters evaluated included volume, sperm count, sperm concentration, motility, morphology, and viability. Baseline characteristics were compared using absolute standardized differences $(7,8)$.

We performed logistic regression models evaluating whether patient or semen characteristics were associated with the use of ART prior to evaluation at a male fertility subspecialty clinic. Hosmer-Lemeshow goodness-of-fit tests were conducted to verify the appropriateness of each model. Variables included in the model were selected a priori and included patient and partner age, year of visit, duration of infertility, and semen analysis parameters.
In the primary analysis, patient and partner age, year of visit, duration of infertility were modeled as continuous terms while semen analysis parameters were modeled as categorical terms, based on the 2010 World Health Organization reference values (9).

We also conducted sensitivity analyses: (I) we modeled interval semen parameters (count, concentration, and motility) as continuous terms; (II) year of visit was modeled as a categorical term based on change in coverage under the universal health care system in Ontario, Canada, which occurred in December 2015 (10). Prior to this date, patients had to pay out of pocket for the use reproductive technology. However, we allowed for a 6-month transition prior to and after December 2015 as the change in coverage was expected, and to accommodate wait times for evaluation in our clinic.

All statistical analyses were performed using SAS (version 9.4; SAS Institute, Cary, NC, USA).

\section{Results}

\section{Cobort characteristics}

In the 22-year study period, we identified 8,962 men who presented to a male fertility specialty clinic at a tertiary academic center. Of these, 1,545 (17.2\%) men reported the use of ARTs prior to evaluation in our clinic. Characteristics of these patients are shown in Table 1. Of these patients, $14.2 \%$ reported current use of cigarettes and $10.1 \%$ reported current use of marijuana. In the last 6 months prior to evaluation, $0.5 \%$ and $0.4 \%$ of patients that had tried ARTs reported the use of testosterone supplements and 5-alpha reductase inhibitors.

Of the patients reporting the use of reproductive technology prior to evaluation, 998 and 289 had tried IUI and IVF, respectively, while 258 had tried both IUI and IVF. More than one attempt was reported in 470 (37.2\%) and $154(28.2 \%)$ of men with prior IUI and IVF, respectively. In patients with prior IUI, the number of reported cycles was 1 in $62.6 \%, 2$ in $21.7 \%, 3$ in $9.1 \%, 4$ in $4.0 \%, 5$ in $1.7 \%, 6$ in $0.6 \%$ and 8 in $0.5 \%$. In patients with prior IVF, the number of reported cycles was 1 in $71.8 \%, 2$ in $16.4 \%, 3$ in $7.3 \%, 4$ in $3.1 \%, 5$ in $0.6 \%, 6$ in $0.4 \%$, and 7 in $0.4 \%$.

In men with prior use of ARTs, semen analysis demonstrated that $12.3 \%$ had volume $<1.5 \mathrm{~mL}, 39.0 \%$ had concentration $<15$ million $/ \mathrm{mL}, 42.0 \%$ had count $<39$ million, $86.3 \%$ had motility $<40 \%, 87.4 \%$ had progressive motility $<32 \%, 17.4 \%$ had normal forms $<4 \%$, and $20.7 \%$ 
Table 1 Characteristics of patients presenting to a male fertility specialty clinic

\begin{tabular}{|c|c|c|c|}
\hline Characteristic & $\begin{array}{l}\text { No prior use of ART } \\
\qquad(n=7,417)\end{array}$ & $\begin{array}{l}\text { Prior use of ART } \\
\qquad(n=1,545)\end{array}$ & Absolute standardized difference \\
\hline Age [years, median (IQR)] & 36 [32-41] & $37[34-41]$ & 0.08 \\
\hline Ethnicity [n (\%)] & & & 0.14 \\
\hline Caucasian & $3,143(42.4)$ & $651(42.1)$ & \\
\hline Hispanic & $156(2.1)$ & $20(1.3)$ & \\
\hline Indo-Canadian & $219(3.0)$ & $57(3.7)$ & \\
\hline Visit year [n (\%)] & & & 0.09 \\
\hline $1995-1999$ & $31(0.4)$ & $3(0.2)$ & \\
\hline 2000-2004 & $419(5.6)$ & $111(7.2)$ & \\
\hline 2005-2009 & $1,503(20.3)$ & $280(18.1)$ & \\
\hline 2010-2017 & $5,464(73.7)$ & $1,151(74.5)$ & \\
\hline Duration of infertility [years, median (IQR)] & $2.0(1.0-4.0)$ & $2.1(1.5-5.0)$ & 0.02 \\
\hline Coital frequency per month [median (IQR)] & $8[4-10]$ & $6[4-10]$ & 0.26 \\
\hline 5-alpha reductase inhibitor & $35(0.5)$ & $6(0.4)$ & 0.01 \\
\hline
\end{tabular}

ART, artificial reproductive technology; IQR, interquartile range.

had viability $<58 \%$ (Table 2).

\section{Predicting use of prior reproductive technology}

Younger male age [adjusted odds ratio (aOR) 0.97 for each 1 year increase in age; $95 \%$ confidence interval (CI), 0.95 to 0.99 ], older female partner age (aOR 1.07 for each 1 year increase in age; $95 \% \mathrm{CI}, 1.04$ to 1.10 ), and year of visit (aOR 1.05 for each 1 year increase; 95\% CI, 1.01 to 1.09 ) were significantly associated with prior use of IUI (Table 3).

Older female partner age (aOR 1.07 for each 1 year increase in age; $95 \% \mathrm{CI}, 1.02$ to 1.12 ) was significantly associated with prior use of IVF, but not male age or year of visit (Table 4).

None of the semen analysis parameters, nor duration of infertility were associated with prior IUI or IVF.

\section{Sensitivity analyses}

When interval semen analyses parameters were modeled as continuous variables, similar to our primary analyses with these variables categorized, none were associated with prior 
Table 2 Semen analysis parameters of patients presenting to a male fertility specialty clinic

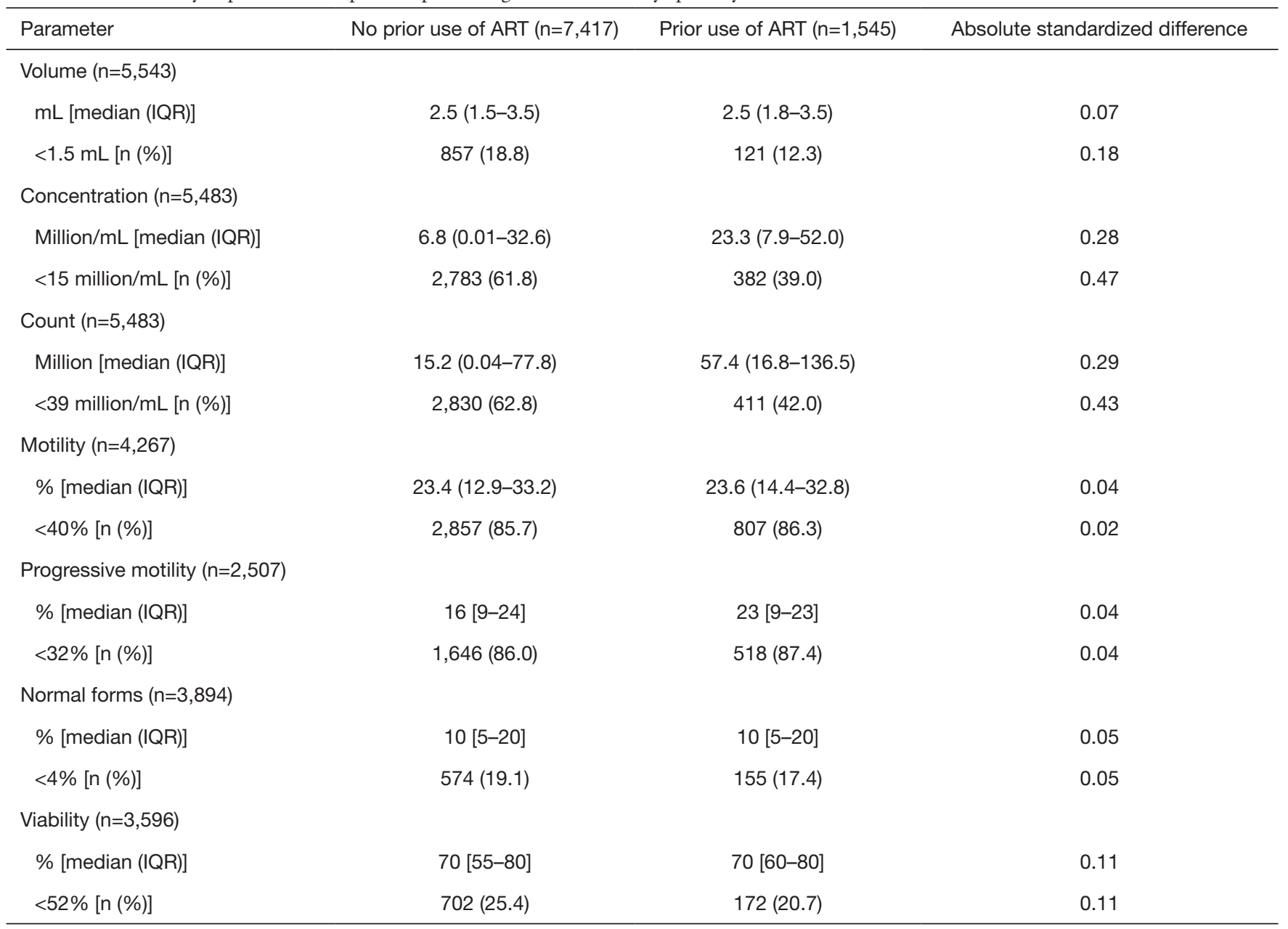

$\mathrm{ART}$, artificial reproductive technology; IQR, interquartile range.

use of IUI or IVF (data not shown).

Dichotomizing year of visit based on the timing of public-funding for ARTs treatments also did not demonstrate an association with prior IUI or IVF (data not shown).

\section{Discussion}

To the best of our knowledge, this is first study to characterize patients using ARTs prior to evaluation with a male fertility specialist. Given the increasing use of ARTs, these findings help us understand the potential role of a male fertility specialist in counseling infertile men.

A review by Ombelet et al. evaluated sperm criteria associated with IUI success (3). In the 55 studies included in their review, the most common sperm parameters examined were the inseminating motile count, morphology, total motile sperm count in the native sperm sample, and total motility in the native sperm sample. Although different thresholds for these parameters have been described, they have been all associated with IUI success (3). Similarly, sperm motility and morphology have also been associated with success of IVF $(4,5)$. Given these data, optimizing semen quality is desirable prior to attempting the use of reproductive technology.

We found that some patients with prior use of ARTs had reversible lifestyle factors associated with sperm quality, such as smoking cigarettes or marijuana. In a cohort study by Kunzle et al., semen analysis data was compared between 655 smokers and 1,131 non-smokers; sperm concentration, count, total motile sperm, and morphology were found to be significantly reduced in those with a reported history of 
Table 3 Multivariable model predicting use of prior intrauterine insemination in men presenting to a male fertility specialty clinic

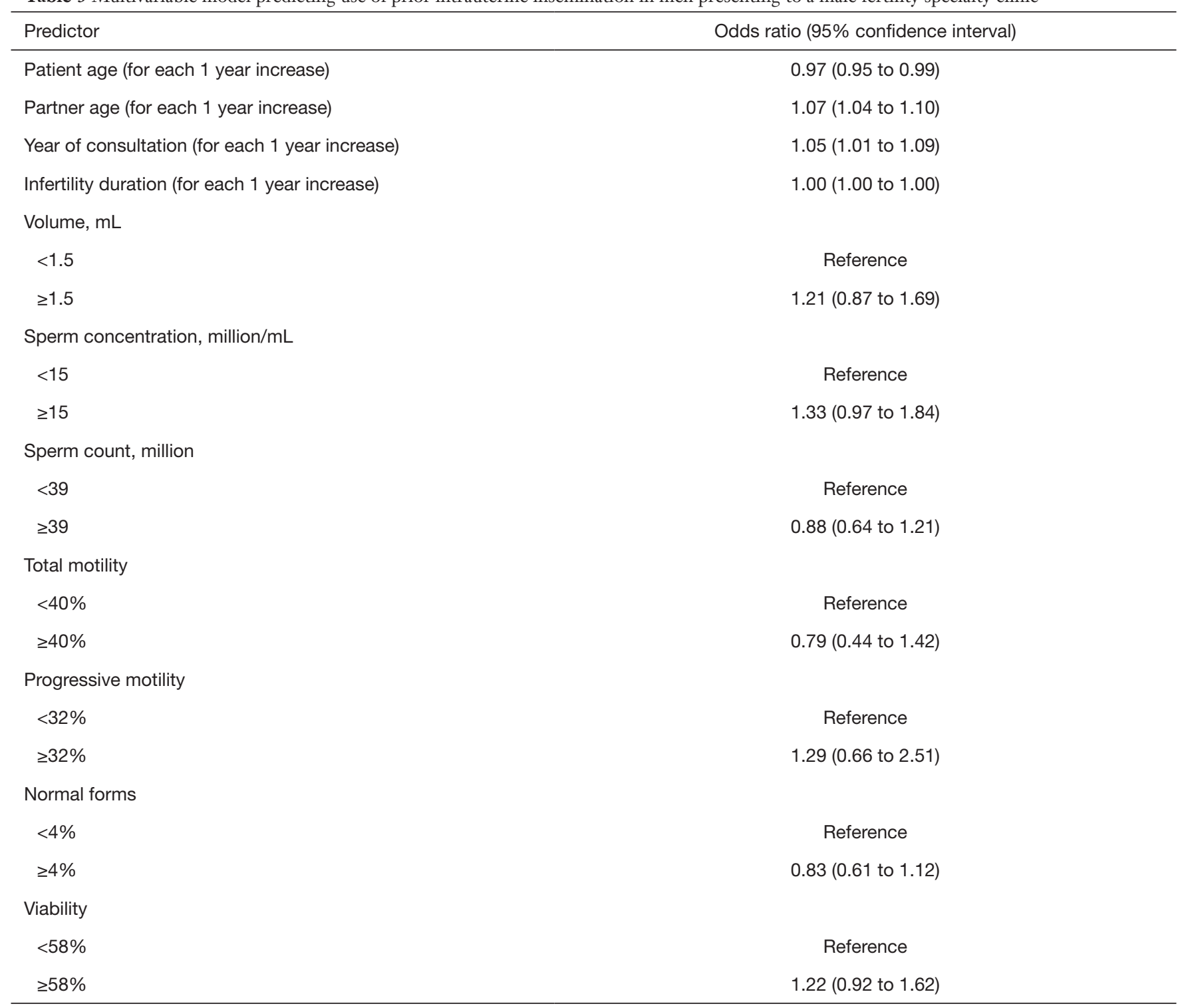

cigarette smoking (11). Another study of 1,215 healthy men found that $45 \%$ had smoked marijuana in the 3 months prior, and those that smoked more than once per week had significantly reduced sperm concentration and total sperm count compared to those that reported no use. Interestingly, there were no significant differences in semen parameters between those that smoked marijuana less than once per week compared to those that reported no use (12). In our study, we found that cigarette smoking and marijuana use were not uncommon among men with use of ARTs prior to evaluation with a male fertility specialist. Furthermore, we found that some patients with prior use of ARTs reported the use of testosterone and 5-alpha reductase inhibitors, both of which have been shown to be associated with adverse semen parameters $(13,14)$. These findings highlight the potential role of a male fertility specialist to appropriately counsel patients on reversible factors to improve semen quality, and thereby potentially improve outcomes when using ARTs. Although such counselling can also be provided by health care providers other than male fertility specialists, the relatively similar proportions of patients with these reversible factors in patients with and without prior use of reproductive technology suggest that any such counselling that may have been provided by other 
Table 4 Multivariable model predicting use of prior in-vitro fertilization in men presenting to a male fertility specialty clinic

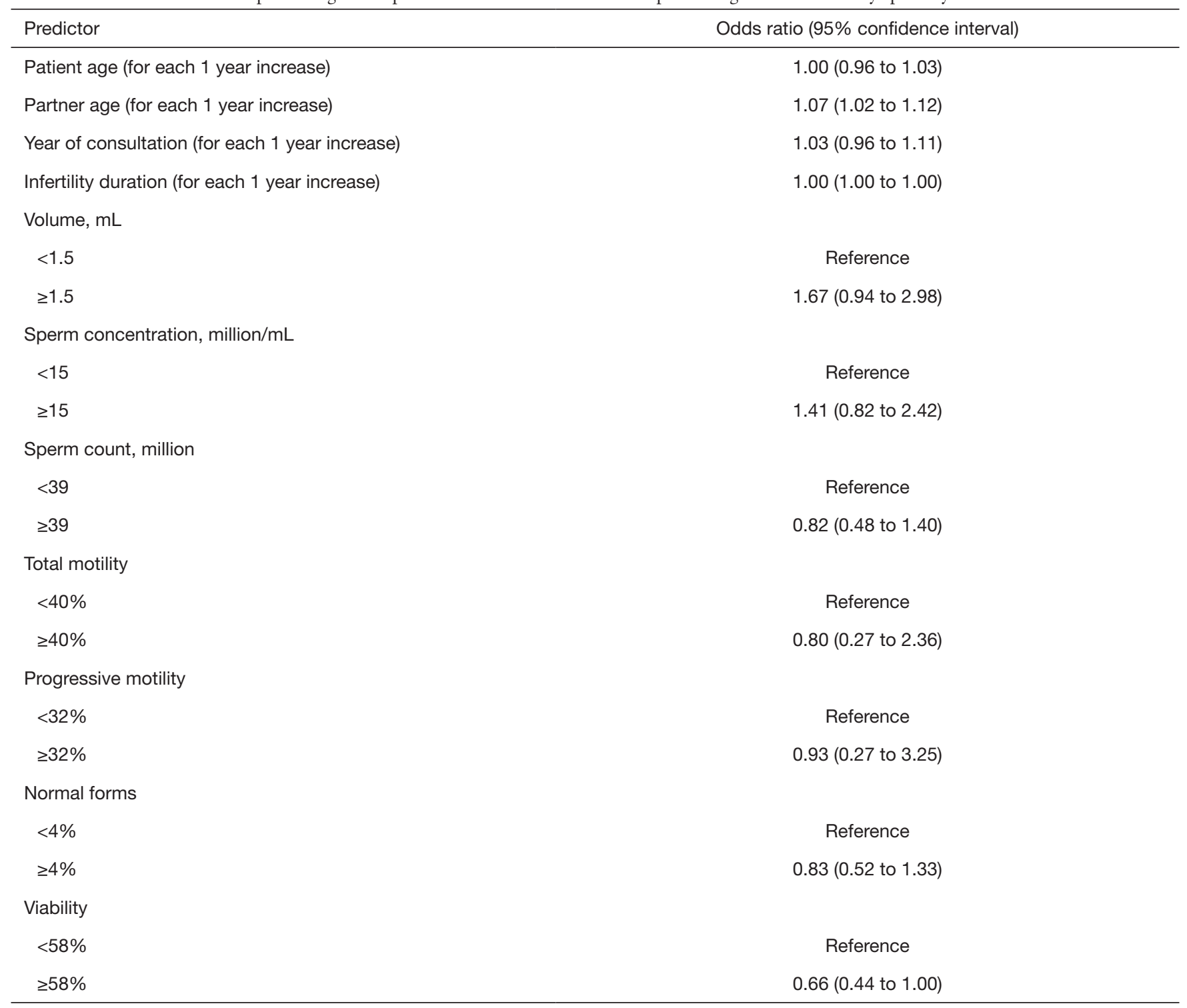

health care providers may not have been very effective. Certainly, further research is needed to understand how male fertility specialists and other health care providers can effectively counsel infertile men to modify reversible factors to improve semen quality.

Our study found that the majority of patients were referred to a male fertility specialist after 1 attempt of IUI and/or IVF. However, several patients were referred after 4 or more attempts. This is an important consideration as studies have shown limited value of many repeated attempts. A prospective cohort study of couples recruited from 8 reproductive endocrinology clinics found that couples using up to 3 cycles of IUI or 2 cycles IVF were more likely to achieve pregnancy; however, further attempts were not associated with increased likelihood of pregnancy, suggesting that there is a marginal benefit obtained with many repeated attempts (15). Another study used data from UK Human Fertilisation and Embryology Authority and found that the cumulative likelihood of success increased modestly after 3 attempts, and minimally after 6 attempts of IVF (16). The male fertility specialist can therefore provide patients with information on the value of repeated attempts to moderate patient expectations and potentially reduce expenses. 
When determining which factors were associated with prior use of ARTs, we found that older female partner age was significantly associated with both prior use of IUI and IVF. In our Canadian publicly funded health-care system, this finding is not surprising given the delay that patients can experience in waiting for a consultation appointment with a male fertility specialist. Whether this finding applies in areas that include privately funded healthcare options remains unknown. Although patients with prior use of reproductive technology were more likely to have normal semen concentration, count, and viability, none of these semen parameters were associated with prior use of reproductive technology after accounting for partner age, suggesting that partner age is the strongest driving factor. Finally, we did not see an association between the time period related to public-funding of reproductive technology and its prior use before evaluation by a male fertility specialist. It would not be unexpected that more patients would seek evaluation prior to attempting use of reproductive technology to optimize their single funded cycle. In a post-hoc analysis, $14.5 \%$ of patients reported use of prior reproductive technology after public funding, reduced compared to $17.6 \%$ in the period prior. This difference was not statistically significant in the multivariable model, but may become more pronounced with time.

Some limitations of our study merit emphasis. Our study relied on semen analysis parameters obtained after consultation at our male fertility specialty clinic and we could not obtain information about the patient's semen analysis parameters prior to the use of reproductive technology. However, it is expected that the semen analysis parameters obtained after consultation are reflective of the patient's prior semen quality. Furthermore, we were unable to identify the proportion of men succeeding with reproductive technology, without consultation by a male fertility specialist. Identifying such patients may help triage patients that will benefit most from consultation with a male fertility specialist. Despite these limitations, our study is the first to characterize patients using ARTs before consultation with a male fertility specialist and helps us understand the potential role of the male fertility specialist in evaluating and counseling these patients.

\section{Conclusions}

While urologists have a significant role in the investigation, management and counseling of couples with infertility, the prior use of ARTs is common among men presenting for an initial evaluation at a male infertility specialty clinic. Older female partner age was associated with higher use of ARTs prior to evaluation. As women become older, their reproductive potential declines (the so called "biological clock") and our study suggests that the fertility centers or/and the couples are more likely to proceed with ARTs if they are faced with this "biological clock". The male fertility specialist may have a role in counselling patients on their likelihood of success with reproductive technology and may provide recommendations to potentially increase the patient's chance of success, reduce financial burden and optimize resource utilization in our healthcare system.

\section{Acknowledgements}

The authors thank Susan Lau for assistance in obtaining the data and facilitating submission of the manuscript.

\section{Footnote}

Conflicts of Interest: The authors have no conflicts of interest to declare.

Ethical Statement: The research was approved by the Research Ethics Board at Mount Sinai Hospital, Toronto, Canada (No. 14-0342-E).

\section{References}

1. Mascarenhas MN, Flaxman SR, Boerma T, et al. National, regional, and global trends in infertility prevalence since 1990: a systematic analysis of 277 health surveys. PLoS Med 2012;9:e1001356.

2. Thonneau P, Marchand S, Tallec A, et al. Incidence and main causes of infertility in a resident population $(1,850,000)$ of three French regions (1988-1989). Hum Reprod 1991;6:811-6.

3. Ombelet W, Dhont N, Thijssen A, et al. Semen quality and prediction of IUI success in male subfertility: a systematic review. Reprod Biomed Online 2014;28:300-9.

4. Kruger TF, Menkveld R, Stander FS, et al. Sperm morphologic features as a prognostic factor in in vitro fertilization. Fertil Steril 1986;46:1118-23.

5. Mahadevan MM, Trounson AO. The influence of seminal characteristics on the success rate of human in vitro fertilization. Fertil Steril 1984;42:400-5.

6. Bhatti T, Baibergenova A. A comparison of the cost- 
effectiveness of in vitro fertilization strategies and stimulated intrauterine insemination in a Canadian health economic model. Bhatt T 2008;30:411-20.

7. Austin PC. Using the standardized difference to compare the prevalence of a binary variable between two groups in observational research. Commun Stat Simul Comput 2009;38:1228-34.

8. Tritchler D. Interpreting the standardized difference. Biometrics 1995:351-3.

9. Castle SM, Cooperberg MR, Sadetsky N, et al. Adequacy of a single 24-hour urine collection for metabolic evaluation of recurrent nephrolithiasis. J Urol 2010;184:579-83.

10. Ontario Ministry of Health and Long-Term Care. Ontario's Fertility Program. 2015. Available online: http:// health.gov.on.ca/en/pro/programs/fertility/

11. Kunzle R, Mueller MD, Hanggi W, et al. Semen quality of male smokers and nonsmokers in infertile couples. Fertil Steril 2003;79:287-91.

Cite this article as: Nayan M, Punjani N, Grober E, Lo $\mathrm{K}$, Jarvi K. The use of assisted reproductive technology before male factor infertility evaluation. Transl Androl Urol 2018;7(4):678-685. doi: 10.21037/tau.2018.06.08
12. Gundersen TD, Jørgensen N, Andersson AM, et al. Association between use of marijuana and male reproductive hormones and semen quality: a study among 1,215 healthy young men. Am J Epidemiol 2015;182:473-81.

13. Samplaski MK, Lo K, Grober E, et al. Finasteride use in the male infertility population: effects on semen and hormone parameters. Fertil Steril 2013;100:1542-6.

14. Liu PY, Swerdloff RS, Christenson PD, et al. Rate, extent, and modifiers of spermatogenic recovery after hormonal male contraception: an integrated analysis. Lancet 2006;367:1412-20.

15. Smith JF, Eisenberg ML, Millstein SG, et al. Fertility treatments and outcomes among couples seeking fertility care: data from a prospective fertility cohort in the United States. Fertil Steril 2011;95:79-84.

16. Smith AD, Tilling K, Nelson SM, et al. Live-birth rate associated with repeat in vitro fertilization treatment cycles. JAMA 2015;314:2654-62. 